\title{
Image diagnosis of Trigeminal Neuralgia caused by Vascular Compression
}

\author{
Moududul Haque ${ }^{1}$, AKM Tarikul Islam², Asifur Rahman³, Sudipta Kumar Mukharjee ${ }^{4}$, \\ ATM Mosharef Hossain ${ }^{5}$
}

Conflict of interest: There is no conflict of interest relevant to this paper to disclose.

Funding Agency: was not funded by any institute or any group.

Contribution of Authors: Principal Investigator and Manuscript preparationData collection-

Scalp block with anaesthesia-

Editorial formatting-

Copyright: @2020bang.BJNS published by BSNS. This article is published under the creative commons CC-BY-NC license. This license permits use distribution (https: //creative commons. orgf/licences/by-nc/4-0/) reproduction in any medium, provided the original work is properly cited and is not used for commercial purposes.

Received: 02. 11.19

Accepted: 16.02 .20

\begin{abstract}
:
Trigeminal Neuralgia (TGN) is a disease frequently encountered by the neurologists and neurosurgeons. The typical pain of TGN is lancinating in nature in one side of face along the distribution of Trigeminal nerve. Pain is sharp shooting and periodic in nature, aggravated by various factors, like eating, talking, laughing. A typical trigeminal neuralgia is caused by compression to the Root exit zone (REZ) by superior cerebellar artery (SCA), aberrant loop of Antero inferior cerebellar artery (AICA), dolichobasilar artery or a large sized vein. However facial pain mimicking TGN may occur by a tumor, plaque of Multiple sclerosis or may be idiopathic.
\end{abstract}

So this is very important to know the cause of TGN/ facial pain for planning of specific treatment.

Most of the patients remain pain free by medical management by using Carbamazepine, oxycarbazepine, Pregabalin, Gabapentin, Clonazepam etc. But medically refractory TGN can be treated by Microvascular decompression (MVD) with significantly satisfactory results if the cause is by vascular compression. However during MVD no significant blood vessels are seen and surgery become failed.

Clinical examination and evaluation is very important, however to become confirmed about the pathogenesis needs imaging of brain. MRI of brain can differentiate any tumor or Plaque of MS. But conventional MRI images can not show us clearly the blood vessel causing TGN.

Conventional MRA could show the blood vessels, which might be causing compression to REZ but does not confirm the fact. Constructive interference in steady state (CISS) MR images were evaluated in our 15 cases to find neurovascular relationship at the REZ. In 11 cases CISS images showed excellent visualization of fifth nerve and blood vessels causing compression to REZ in all case of TGN who underwent Microvascular decompression (MVD) with excellent result. In 2 patients we depended on clinical findings and T2 MR Images. In one of them we could find Offending vessels who were also improved clinically. In one case we did not find any significant offending vessel except a small vein and this patient did not show any improvement. In rest two cases, in one patient CISS showed a tiny vessel and after MVD the patient did not improve. In one patient no significant vessels were not seen in CISS images. We did not go for MVD for this case.

So the pre-operative CISS MR images are more precise to show the neurovascular relationship and determine the offending blood vessel causing TGN. Thus we can avoid an unnecessary MVD.

Key words: TGN, CISS, MVD.

Bang. J Neurosurgery 2020; 10(1): 3-8

\section{Introduction:}

Trigeminal Neuralgia can be caused by vascular compression to the Root exit zone (REZ) of trigeminal nerve where the nerve is not covered by myelin sheath for few millimeters.
TGN is a peculiarly painful paroxysmal disorder with an annual incidence rate of 4.3 per 100000 population with a male female ratio of $1: 1.17^{7}$ Microvascular decompression (MVD) is frequently used for cure of TGN by displacing the offending vessel from the REZ.

1. Associate Professor, Department of Neurosurgery, BSMMU, Dhaka

2. Associate Professor, Department of Neurosurgery, BSMMU, Dhaka

3. Associate Professor, Department of Neurosurgery, BSMMU, Dhaka

4. Associate Professor, NINS, Dhaka

5. Professor \& Chairman, Department of Neurosurgery, BSMMU, Dhaka 
However MVD is not always curative. The success rate is $84 \%{ }^{15}$. Sometimes any definite offending vessel can not be seen during an MVD. This is because of inappropriate evaluation or lack of proper imaging study. The post operative recurrence rate is significantly high when a small unnamed artery or vein is thought to be responsible for $\mathrm{TGN}^{3}$. Each year thousands of patients undergo surgery for TGN in the United States, with an estimated cost of $\$ 100$ million with excellent result in most of the cases but some patients do not have any pain relief ${ }^{18}$. Results of MVD are poor when surgery discloses no vascular compression or only small venous contact with the nerve ${ }^{17}$.

Conventional MRI in T1 or T2 images or MRA can not clearly delineate the offending blood vessel causing TGN although they can show clearly any tumor causing the problem (Fig 1, 2, 3).

$\mathrm{MRI}$ in Constructive interference in steady state (CISS) protocol can clearly show contrast between the nerve, blood vessels and CSF. So, by studying TGN by CISS MR Images can help us select patient of true TGN caused by vascular compression and thus increase the good outcome of surgery and avoid unnecessary surgery.

\section{Material and Methods:}

Our study included 15 patients (aged $45-73$ years, 3 females 12 males). All these patients were clinically showed typical features of trigeminal neuralgia and were treated with carbamazepine and other drugs. All the patients underwent conventional MRI of brain to rule out any tumor or demyelinating diseases or any other pathology. All these patients were refractory to medical management.

For further evaluation we used CISS MR Images. All images were done in single center using a 1.5 tesla MRI machine and confirmed by a neuroradiologist. Axial images of $1 \mathrm{~mm}$ thickness in continuous slices at cerebello pontine angle were reviewed to confirm the relationship of offending vessels and the nerve.

\section{Results:}

CISS MR Images could show the offending vessels more precisely because this can delineate an excellent contrast between the nerve and CSF (Figure 1).

Furthermore CISS can show large petrosal veins which also may be the cause of TGN. In our 11 cases CISS images showed excellent visualization of fifth nerve and blood vessels causing compression to REZ in all case of TGN. Who underwent Microvascular decompression( MVD)with excellent result.

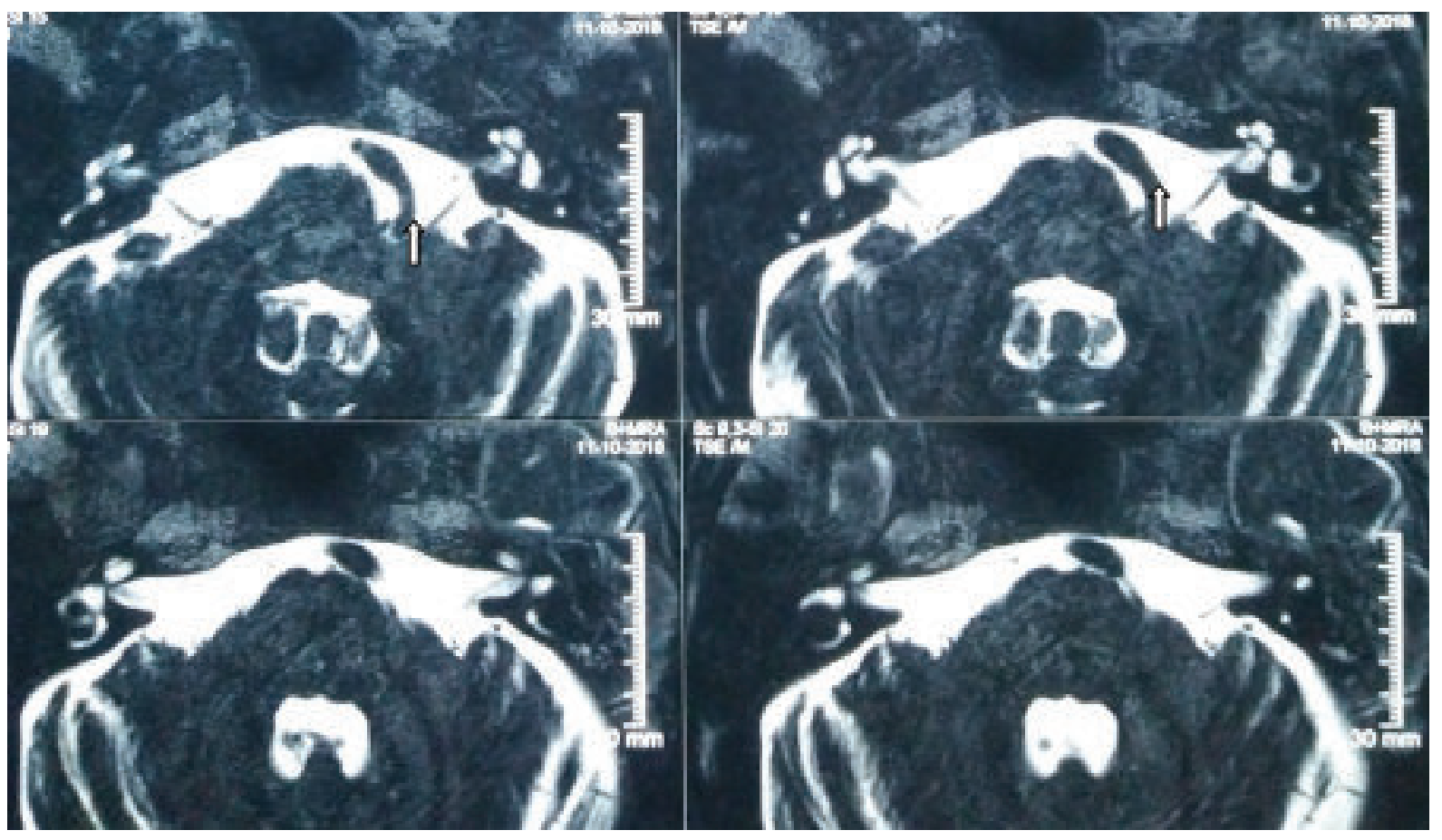

Fig.-1: CISS MR image showing clearly the offending vessel. Shown by white arrows 
We used a lateral park bench position and a key hole craniectomy and surgery was done under operating microscope in high magnification and we used tiny pieces of surgical sponge to decompress in all our patient who underwent MVD.

In most cases, the offending vessel was Superior Cerebellar Artery (SCA). In one case it was a dolichobasilar artery and in one it was a loop of Antero inferior cerebellar artery (AICA). In most of the cases the Trigeminal nerve was found pushed, thin and slightly reddish in appearance during surgery. In our series, 11 patients who were evaluated by CISS showed excellent result. Trigeminal pain improved from the next day of surgery. In the patients TGN caused by a tumor is readily be diagnosed by an MRI (Figure 2).

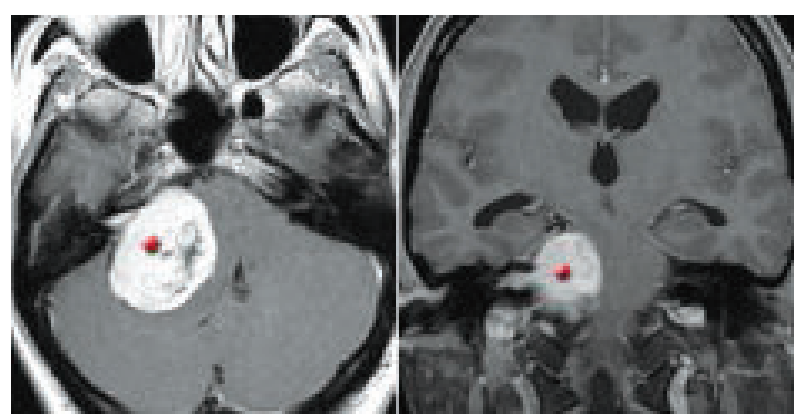

Fig.-2: CPA tumor compressing fifth nerve.
MRI of tumor ). In the patients who had TGN due to vascular compression, conventional MR image can not clearly show the offending vessels ( Figure3).

TGN MRI). However CISS MR images could clearly showed the offending vessels compressing the Trigeminal nerve at REZ (figure 4,5,6,7).

Offending vessels were found in all these cases (Figure 8,9 Per operative picture ) and all the patients improved after MVD. Two patient underwent MVD on the basis of MRI images and clinical findings, in one patient the offending vessel was seen during surgery and recovered very well. But in one patient, we could not find any significant offending vessels (figure 10).

In two cases CISS showed a very thin vessel in one ( figure $11 \mathrm{CISS}$ thin vessel) and no vessel in one (Figure 12 CISS showing no vessel)

MVD was done in the patient where CISS showed a tiny vessel, a tiny vein was found during surgery. This patient did not improve after surgery. In the patient in whom CISS image did not show any offending vessel and we did not go for MVD in this case. In our cases the most common offending vessel found during surgery were SCA, in one case it was a loop of AICA and in one case a dolicho basilar artery was itself compressing the nerve. In all cases moderate to severe displacement of the Trigeminal nerve was seen.

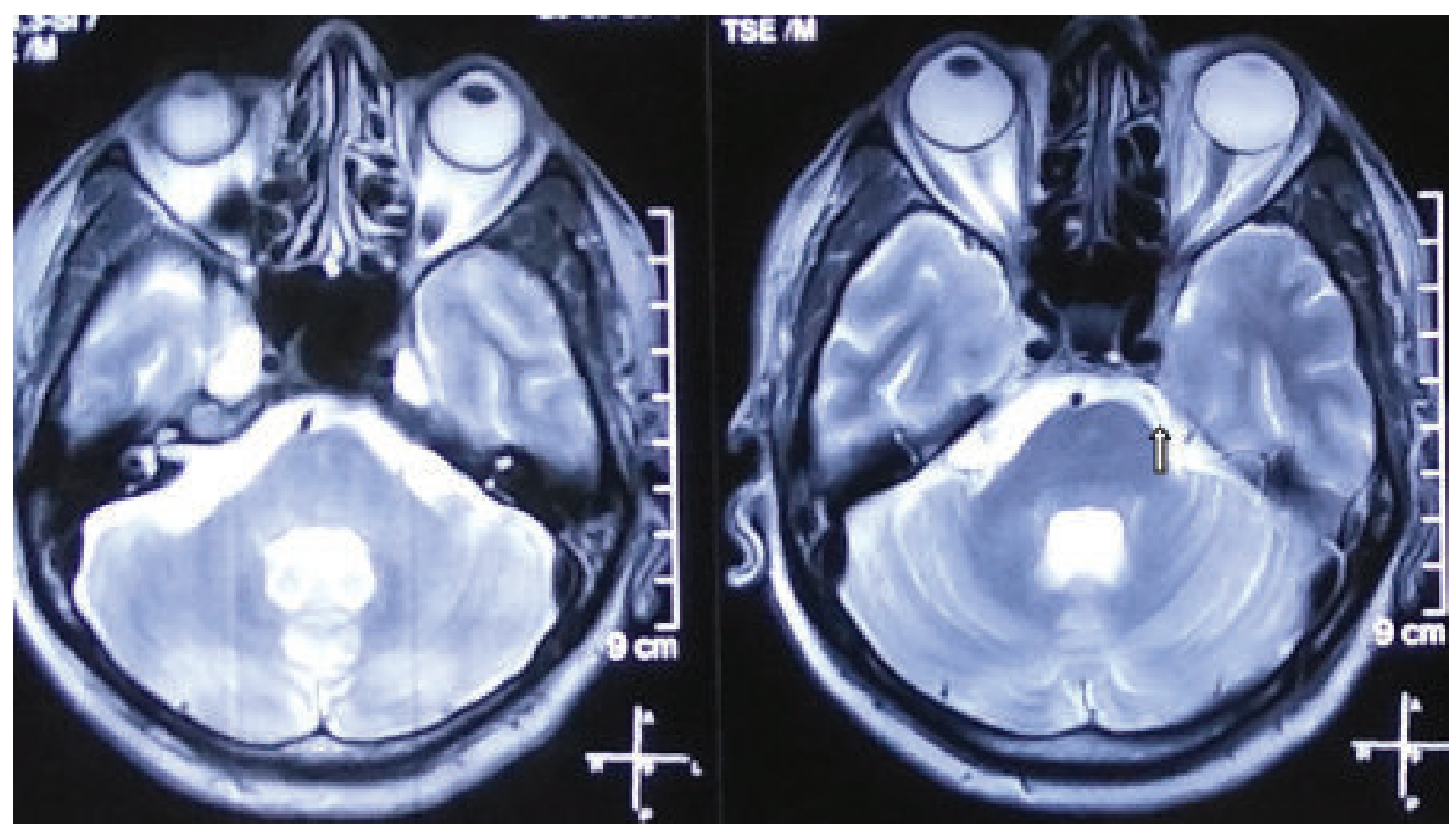

Fig.-3: The delineation of offending vessel is not convincingly clear, white arrow. 

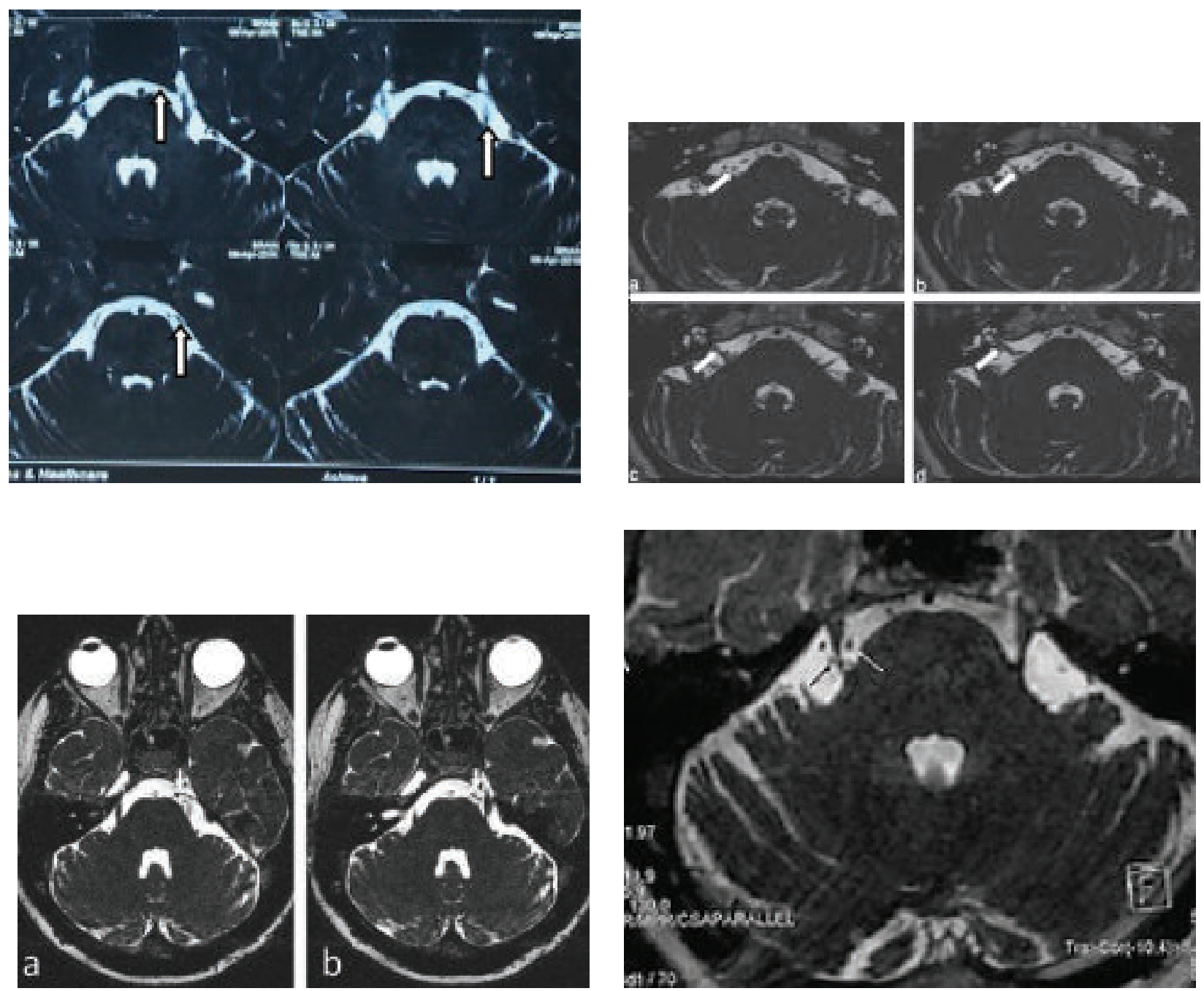

Fig.-4,5,6,7:
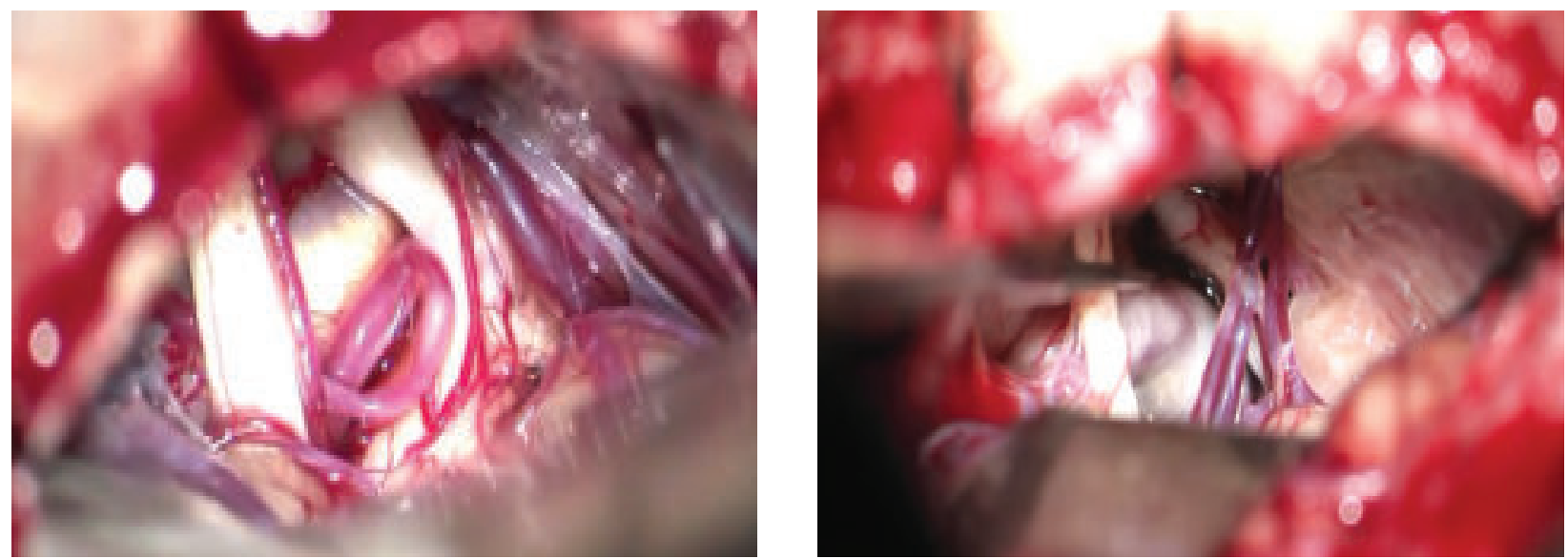

Fig.-8: shows compression by SCA

Fig.-9: showing compression by a dolichobasilar artery 




Fig.-10: No significant offending vessel seen during surgery

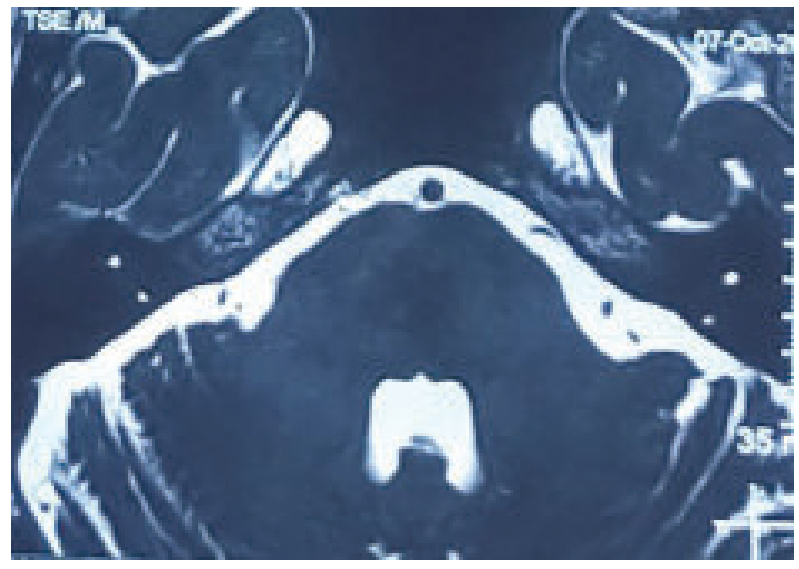

Fig.-11: Thin vein is seen not compressing the nerve on the affected side

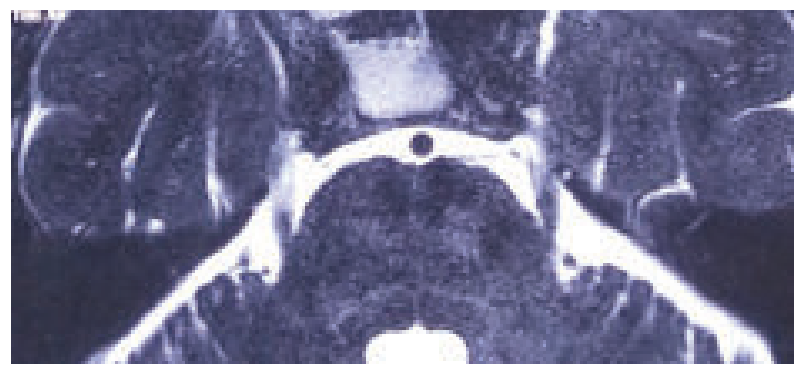

Fig.-12: No offending vessels are seen in CISS

\section{Discussion:}

TGN is a peculiarly painful paroxysmal disorder with an annual incidence rate of 4.3 per 100000 population with a male female ratio of $1: 1 \cdot 17^{7}$. Most commonly the TGN is caused by compression of Trigeminal nerve at its REZ by blood vessels ${ }^{16}$. This was first described by Dandy 6 .

Conventional T1 and T2 MR Images can exclude tumors or multiple sclerosis plaques in the patients who manifest as TGN. Therefore these conventional images are indispensable for routine preoperative assessment of TGN. However the main goal of pre operative images is to identify the neurovascular relationships at REZ. Generally high resolution MR imaging is implemented to identify the neurovascular contact of the TGN and exclude other pathology of TGN, however, this neurovascular contact has also been reported in normal patients and also in unaffected side in TGN patients. The vascular contact to REZ of TGN should be at the region of the trigeminal nerve where there is a lack of myelin sheath to cause TGN. In these case MVD is considered most effective treatment ${ }^{14}$. The maximum diameters of the offending vessels are less than $2 \mathrm{~mm}^{19}$. The slice thickness of $3 \mathrm{~mm}$ in conventional T1 and T2 weighted imaging may miss vascular compression at the REZ because of partial volume effect, since the offending artery is small in relation to slice thickness. Therefore these conventional sequences are not sufficiently sensitive to depict such neurovascular relationship ${ }^{11}$. Loss of anisotrophy in trigeminal neuralgia revealed by diffusion tensor imaging ${ }^{4}$. Three dimensional Time of flight (TOF) MR angiography have shown that neurovascular compression (NVC) at the unaffected trigeminal nerve is typically not as severe as at the affected nerve. TGN is associated with atrophy of trigeminal nerve ${ }^{1,2}$, . The most common offending vessels are SCA and AICA In TGN ${ }^{9,10,13}$. as are seen in our cases.

The CISS MR sequence provides a finely detailed cisternographic image in which the brain nerves and vessels appear with low signal intensity and are well delineated with high signal intensity of $\mathrm{CSF}^{5,8}$. The contrast between the hypointense brain, nerves and vessels and hyperintense CSF is excellent. CISS also provide high spatial resolution based on very thin sections with no intervening gaps ${ }^{12}$. CSF movement in CISS can abolish signal loss due to CSF flow in the $\mathrm{CP}$ angle and thus can depict precisely the small arteries and veins. Visualization of Petrosal vein can predict pre operatively can give confidence to the surgeons as injury to petrosal vein may sometimes cause venous complication during surgery. Although petrosal vein sometimes is the offending vessel for TGN.

CISS can precisely delineate the offending vessel in contact with REZ in symptomatic patients and in our cases 11 patients showed definite cause of compression and found clearly during surgery and the 
patients improved after MVD. Very thin vein which are also seen in CISS images are probably not the cause of TGN although patients are symptomatic and should be considered as idiopathic cause and can be treated by other modalities.

\section{Conclusion:}

For pre operative evaluation of TGN, conventional MRI images are important to find out any mass lesion however for neurovascular compression, CISS MR images may be thought as gold standard. So every patients of TGN should be evaluated by CISS which can precisely delineate the offending vessel and at the same time can show no vessels or very tiny veins who may not be the candidate for MVD, thus CISS can prevent the surgeons to avoid unnecessary surgery.

\section{Find unsuccessful MVD from internet}

\section{References:}

1. Anderson V.C, Berryhill P.C, Sandquist M.AClaverella D.P, Nebit G.M and Burchiel K.J. High resolution threedimensional magnatic resonance angiographyand threedimensional spoiled gradient-racalled imaging in the evaluation of neurovascular compressionin patients with trigeminal neuralgia: a double-blind pilot study. Neurosurgery.58:666-673, 2006

2. Antony G, Di Pasquale A, Cruccu G, Truini A, Morino S, Saltelli G. Magmnatic resonance imagingcontribution for diagnosing symptomatic neurovascular contactin classical trigeminal neuralgia: a blinded case control study and meta analysis. Pain. 155:1464-1471, 2014

3. Barker FZ, Jannetta PJ, Bissonette DJ, Shields PT, Larkins MV, Jho HD: Microvascular decompression for hemifacial spasm. J Neurosurg 82:201-210, 1995

4. C Herweh, B Kress, D Rasche, V Tronnier, J Tröger. Loss of anisotropy in trigeminal neuralgia revealed by diffusion tensor imaging.Neurolgy.68:776-778, 2007

5. Casselman JW, Kuhweide R, Deimling M, Ampe W, Deheans I, Meeus L.Constructive interfence in steady state-3DFT MR imaging of the inner ear and cerebellopontine angle. AJNR AM JNeuroradiol. 14:47-57,1993

6. Dandy W. E. An operation for the cure of tic douloureux: partial section of sensory root of Pons. Arch Surg.18:687734, 1929
7. David Bowsher. Trigeminal Neuralgia: An Anatomically Oriented Review. Clinical Anatomy 10:409-415,1997

8. Haacke EM, Wielopolski PA, TKach JA, Modic MT. Steadystate precission imaging in the presence of motion: Application for improved visualizationof cerebrospinal fluid. Radiology. 175:545-552, 1990

9. Hardy DG, Rhoton AL.Microvascular relationship of the superior cerebellar artery and trigeminal nerve. J Neurosurg 49:669-678, 1978

10. Haines SJ, Jannetta PJ, Zorub DS. Microvascular relations of the trigeminal nerve: an anatomical study with clinical correlation. J Neurosurg 52: 381-386, 1980

11. Hutchins LG, Harnsberger HR, Jacobs JM, Apfelbaum RI. Trigeminal neuralgia(tic douloureux): MR Imaging assessment. Radiology. 175:837-841, 1991

12. Iwao YAMAKAMI, Eiichi KOBAYASHI, Shinji HIRAI and Akira YAMAURA. Preoperative Assessment of trigeminal Neuralgia and Hemifacial Spasm Using Constructive Interference in Steady State-Three dimensional Fourier Transformation Magnetic Resonance Imaging. Neurol Med Chir (Tokyo).40:545-556, 2000

13. James E. Coad B.S, Jonathan D., Wirtschafter M.D. , Stephen J. Haines M.D., Roberto C. Herds M.D. and Theresa Perrone M.D. Treatment of trigeminal neuralgia by micro-operative decompression, in Youmans JR (ed): Neurological Surgery. Philadelphia, WB Saunders, 3928-3942, 1990

14. Love S, Gradidge T, Coakham HB. Trigeminal neuralgia: pathology and pathogenesis. Brain. 124:247-260, 2001

15. Lovely Tj: Efficacy and complications of microvascular decompression: A review. NeurosurgeryQuarterly 8:92106,1998

16. Nurimikko $\mathrm{T} \mathrm{J}$ and Eldridge $\mathrm{P}$ R. Trigeminal Neuralgiapathophysiology, diagnosis and current treatment. Br.J.Anaesth. 87:117-132,2001

17. Piatt JH Jr, Wilkins $\mathrm{RH}$ : Treatment of tic douloureux and hemifacial spasm by posterior fossa exploration: Theraputic implications of various neurovascular relationships. Neurosurgery 14: 462-471, 1984

18. Pollock B.E and Ecker. R.D. A postoperative study of trigeminal neuralgia surgery. Clin J. Pain, 21:317-322, 2005

19. Yasargil MG. Microsurgery, Vol1. New York, ThiemeStratton, 128-143, 1984 